\title{
Traditional Chinese Medicine Strategy for Patients with Tourette Syndrome Based on Clinical Efficacy and Safety: A Meta- Analysis of 47 Randomized Controlled Trials
}

\author{
Na Wang $\mathbb{D}^{1}{ }^{1}$ Dong-dong Qin, ${ }^{2}$ Yu-huan Xie, ${ }^{2}$ Xin-chen $W u,{ }^{3}$ Ding-yue Wang, ${ }^{3}$ Hang-Yang, ${ }^{3}$ \\ Xiao-xuan Li, ${ }^{3}$ Lei Xiong $\mathbb{D}^{1,2}$ and Jing-hong Liang $\mathbb{B}^{4}$ \\ ${ }^{1}$ School of Basic Medical Science, Shanghai University of Traditional Chinese Medicine, Shanghai 201203, China \\ ${ }^{2}$ School of Basic Medical Science, Yunnan University of Chinese Medicine, Yunnan Kunming 650500, China \\ ${ }^{3}$ First Affiliated Hospital, Yunnan University of Chinese Medicine, Yunnan Kunming 650500, China \\ ${ }^{4}$ Department of Maternal and Child Health, School of Public Health, Sun Yat-sen University, Guangzhou 510080, China
}

Correspondence should be addressed to Lei Xiong; xlluck@sina.com and Jing-hong Liang; liangjh78@mail2.sysu.edu.cn

Received 4 October 2020; Revised 23 January 2021; Accepted 8 February 2021; Published 11 March 2021

Academic Editor: Zan Gao

Copyright $\odot 2021 \mathrm{Na}$ Wang et al. This is an open access article distributed under the Creative Commons Attribution License, which permits unrestricted use, distribution, and reproduction in any medium, provided the original work is properly cited.

\begin{abstract}
Objective. Although increasing evidence reveals the efficacy of traditional Chinese medicine (TCM) and its safety on Tourette Syndrome (TS) patients, whether TCM is indeed improving TS remains unclear. The purpose of the current study is to perform a meta-analysis to evaluate the efficacy and safety of TCM on treating TS patients. Method. An elaborate search strategy was conducted based on several databases including Medline, Embase, Cochrane, Web of Science, CINAHL, CBM, VIP, CNKI, and Wanfang Data in order to identify the relevant randomized controlled trials (RCTs) from their inception to as late as May 1st, 2020. General information and data needing analysis were extracted simultaneously for the necessity of various analyses such as descriptive analysis and metaquantitative analysis. Results. Forty-seven trials with 5437 TS patients in total were eventually included according to our criteria. All trials were conducted in China, and the publication years ranged from 2004 to 2017 . In terms of clinical efficacy, clinical symptoms of patients with TCM were more likely to be improved compared with the control group (odds ratio, $\mathrm{OR}=-1.29,95 \%$ confidence interval, $\mathrm{CI}:-2.54$ to $-0.06, I^{2}=0.00 \%$ ). As to the outcome of recurrence rate, the pooled results revealed that the TCM group was more inclined to stabilize the recurrence ( $\mathrm{OR}=0.44,95 \%$ CI: 0.24 to 0.78 , $\left.I^{2}=0.00 \%\right)$. Similar results were observed in adverse reaction ( $\mathrm{OR}=0.32,95 \% \mathrm{CI}: 0.24$ to $\left.0.43, I^{2}=32.90 \%\right)$. Conclusion. The results of our study recommend applying TCM to treat TS patients for better efficacy and safety. Results need to be interpreted cautiously due to certain limitations in our study.
\end{abstract}

\section{Introduction}

Tourette Syndrome (TS), a type of neurodevelopment disturbance, is commonly characterized by the occurrence of sudden, brief, intermittent movements or vocalization, with a high incidence rate among young children (three to eight cases out of 1000) [1] and over 1\% globally [2]. Symptoms of TS patients are frequently accompanied by cooccurring disorders, such as attention-deficit/hyperactivity disorder (ADHD), anxiety disorder, obsessive-compulsive disorder, and schizophrenia $[3,4]$. The symptoms and complications of TS will trigger a series of consequences for both the patients and their families, even the society sometimes, with TS patients' life quality harmed, financial burden increased, and social resources consumed $[5,6]$.

In practice, guanfacine and clonidine are recommended as the first choice in the treatment of TS patients, along with atypical drugs functioning as dopamine receptor antagonists, namely, antipsychotics such as tiapride and haloperidol, whose efficacy has been proved in numerous clinical trials $[7,8]$. However, previous researches also indicated that the mentioned drugs may bring serious adverse reactions (ARs) to patients $[9,10]$. Therefore, in the absence of a universally accepted and reasonable treatment and with new clinical 
trials to seek possible drug candidates evolving frequently, there is an urgent need for clinical researchers to figure out a reliable treatment to solve this disorder [11].

Currently, complementary and alternative medicine is arresting more attention for their relatively higher efficacy and safety than traditional first-tier drugs, and some studies even point out that $64 \%$ TS patients have received this kind of relatively effective clinical approach for now [12]. As a branch of alternative medicine and ethnopharmacology that dates back to ancient China, traditional Chinese medicine (TCM) has been widely supported by clinical trials for its efficacy and lower rate of recurrence and undesirable side effects [13]. Developed based on traditional and modern medicine theories, TCM, or TCM-assisted treatments, with obvious advantages of high bioavailability and quick effect, is widely used for treating various clinical diseases and, to some extent, effective in relieving the clinical symptoms, such as primary nephrotic syndrome, poststroke depression, or communityacquired pneumonia [14-16]. Although several trials have examined the effectiveness of TCM in treating TS, there have been few studies to combine the available evidence of trials to explore the potential relationship between TCM and TS in terms of efficacy and safety. Two groups of comparisons were conducted. The first compared the effectiveness of TCM with placebo and Western medicine (WM), the second the effectiveness of combined TCMs with WM. The study, however, only quantitatively analyzed the efficacy of TCM based on limited outcomes and studies [17]. Furthermore, the quality of studies included and insufficient analysis from previous meta-analyses were prone to threaten the conclusion. Accumulating evidence regarding the efficacy and safety of TCM on TS was fragmented and inconsistent. Therefore, we formulate a hypothesis to identify the efficacy and safety of TCM in treating TS patients by maximizing, summarizing, and analyzing the available evidence.

\section{Method}

Our study was performed as instructed by the Preferred Reporting Items for Systematic Reviews and Meta-Analyses (PRISMA) statement [18], the Cochrane Collaboration Handbook recommendations [19]. No ethical approval or patient consent was required in that all analyses were conducted based on previously published studies.

With no language or publication time restrictions, a comprehensive literature search was carried out to identify the relevant randomized controlled trials (RCTs) which investigated the efficacy of traditional Chinese medicine on TS in the following electronic databases: Medline (via PubMed), Embase, Cochrane, Web of Science, CINAHL, Chinese National Knowledge Infrastructure, Wanfang Data, Chinese Scientific Journal database, Chinese Biomedical Literature Database, Chinese Clinical Trial Registry, and clinical trials (http://www.clinicaltrials.gov) from their inception to May $1^{\text {st }}, 2020$.

Through the medical subject headings (MeSH) incorporated with free text terms by using the Boolean logical operators, an exhaustive search was performed, with the following terms: "Tourette Syndrome", "Traditional Chinese medicine", "Drugs, Chinese herbal", "Randomized controlled trial" taken into account. Moreover, we conducted a series of recursive searches as complementary retrieval from top journals (top journals in China: China Journal of Chinese Materia Medica, Journal of Traditional Chinese Medicine, China Journal of Traditional Chinese Medicine and Pharmacy, and China Pharmacy; top international journals: Science China Life Sciences, Frontiers in Pharmacology, Frontiers in Microbiology, International Journal of Biological Macromolecules, and Pharmacological Research), famous publishers, major international conference proceedings, and grey literature ((theses of doctors' and masters' noncommercial bibliography, technical documents (including government reports)) to minimize the loss of omission of suitable articles that meet our inclusion criterion. Bibliography from included studies and similar meta-analyses and systematic reviews were additionally screened for potentially eligible studies. Details of all databases of search strategies were shown in supplement search strategies.

A check was indispensable for the integrity and veracity of studies. All records from the initial search were imported into EndNote X9 (Thomson ISI Research Soft, Philadelphia, Pennsylvania, USA), managing and confirming the above information which was performed concurrently by two independent authors. Discrepancies during this progress were settled by discussion or judged by the third author.

2.1. Eligibility Criteria and Selection Process. Studies were included with the PICOS criteria as follows:

2.1.1. Population. Participants were of any age diagnosed with TS (neurodevelopment disturbance commonly characterized by the occurrence of sudden, brief, intermittent movements or vocalization) by proper medical diagnosis criteria, such as the Diagnostic and Statistical Manual, fourth edition, or the text revision of the fourth edition (DSMV/IV) [20], the Chinese Classification of Mental Disorders (CCMD), whether the patients were diagnosed according to CCMD-2R or CCMD-3.

2.1.2. Interventions. Acceptable treatments included any structured and conceptualized traditional Chinese medicine (TCM), such as Ningdong granule, Qufeng Zhidong recipe. Any CTM in combinatorial or multicomponent was excluded.

2.1.3. Comparators. Studies were included if their comparison groups were set as any placebo such as active Western medicine, nutrient, while the studies were excluded when their control groups (CGs) were conducted in any combination with any placebo.

2.1.4. Outcomes. As an indication of reporting the response rate that both occurred in the treatment and control group, the primary outcome was clinical efficacy (CE) which was measured by a variety of rating clinical instruments. Secondary outcomes were other clinical indications, such as Yale Global Tic Severity Scale (YGTSS) [21], adverse reaction (AR), and TCM Syndrome Score Scale (TCMSSS), a widely used clinical tool designed in a questionnaire and monitored by the Xiangya Hospital. 
2.1.5. Study Design. Only the parallel-group RCTs that have been published with no language restrictions were involved.

As defined in our included and excluded criteria, duplications were removed first. Meanwhile, two authors independently selected the studies by screening their titles and abstracts. Subsequently, full-text reviewing was performed in an effort to retrieve the potentially eligible studies. Inconsistencies that emerged in this part were avoided.

2.2. Data Collection and Quality Assessment. Based on the Cochrane Consumers and Communication Review Group's data extraction template [19], a rigorous process of data inspection was performed to extract the key data of included studies by two authors independently. The following relevant items were collected on the basis of the preelaborated measurement: major author responsible for the study, publication year, total sample size, therapeutic course, various outcomes, area of study, etc.

Two investigators independently applied the Cochrane Risk of Bias tool (ROB) in the evaluation of the quality of each included study [19], which consisted of seven items (random sequence generation, allocation concealment, blinding of participants and personnel, blinding of outcome assessment, incomplete outcome data, selective reporting, and other biases), and each of the items was rated as unknown, low, and high risk of bias, respectively.

2.3. Statistical Analyses. Based on the Cochrane Collaboration Handbook recommendations [22], a conventional pairwise meta-analysis of crossed trials was conducted for each comparison. Firstly, in terms of statistical heterogeneity, $I^{2}$ statistics, whose values were $25 \%, 50 \%$, and $75 \%$, respectively, indicating mild, moderate, and high heterogeneity, were provided to determine whether there was a substantial heterogeneity produced [23]. Secondly, for the outcomes presented as numerical variables, we either extracted their mean difference and standard deviation of the change from baseline or transformed them into a standard format to ensure that our analyses were smoothly performed. Moreover, in the presence of effect sizes referring to continuous outcome, standard mean differences (SMDs) were calculated for each comparison using group (relevant) means and standard deviations (SD) from individual studies. For dichotomous variables, the odds ratio (OR) was used to compute the pooled effect sizes (ESs) for each study with the random-effects model [24], whose $95 \%$ of confidence interval (CI) were calculated along with the two combined ES above as a measurement of estimated uncertainty. Thirdly, if the number of included studies in relevant outcomes is over ten, the comparisonadjusted funnel plot based on it will be drawn to detect the presence of any prominent types of potential bias through intuitive vision, such as publication bias, selective reporting, or other biases. As a quantitative complement for the funnel plot, the Egger test was additionally conducted to observe whether the $P$ value was less than 0.05 [25]. At last, based on various interesting variables, planned random-effects subgroup analyses were conducted to ensure the robustness of the summarized ES and all of them were judged as preestablished concomitant variables. A sequence of areas was taken into consideration in our model shown as follows: course of disease (average course $\leq 3$ years vs. average course $>3$ years); condition of disease (mild vs. moderate-severe); publication year (year $\leq 2010$ vs. year $>2010$ ); therapeutic course (duration weeks $\leq 12$ vs. duration weeks $>12$ ); diagnostic standards (CCMD vs. DSM vs. other criteria); total sample size (sample size $\leq 100$ vs. sample size $>100$ ); region (economically backward vs. developed economy); risk of bias group (high quality vs. low and unclear quality group). Furthermore, metaregression based on the random effects was carried out to explore the sources of existing heterogeneity by adjusting the potentially confounding factors if necessary [23]. The above battery of analyses was performed in STATA, version 15.0 (StataCorp, College Station, TX).

\section{Results}

3.1. Literature Selection and Characteristics of Included Studies. The initial target databases search yielded 807 records while 21 were found by extra manual search, among which 58 articles were removed due to duplication. As a result, the number reduced to 142 according to the title and abstract screen results. After the full-text review, 99 studies were excluded, whose contents were irrelevant as follows. Patients of 24 studies were not diagnosed with TS, and 27 studies were not launched as RCT. Meanwhile, 31 studies did not have an explicit definition on TCM and 6 studies did not report the related outcomes. Furthermore, 11 studies took the TCM in combinatorial. At last, we retrieved 11 studies by hand search and 4 were eligible to be included. In that case, 47 unique trials [26-72] in total were finally included in our study. Details of the selection process are displayed in Figure 1.

All 47 trials were conducted in China with publication years ranging from 2004 to 2017. Altogether, 3231 participants were randomized to the TCM group while the control group comprised 2206 participants with an overall median age of 8.17 years old. The proportion of the male patients $(N=3549)$ was significantly higher than female patients $(N=1228)$ (two studies were not reported). Participants recruited and recorded in all trials met at least one standard diagnostic criterion, such as DSM-IV $(N=30)$, CCMD $(N=10)$, or other clinical diagnostic instruments $(N=7)$. The disease course of patients with TS ranged from 1 to 9 years old, their therapeutic course 3 to 24 weeks. Demographic characteristics of the 47 trials are described in Table 1.

3.2. Quality of Included Studies. A relatively low or unclear risk of bias was obtained in 40 of the included trials while 7 studies were categorized as high risk of bias $[36,37,39,41$, $62,70,71]$. Sufficient generation of random sequence was observed in all 47 trials whereas few of them had given their allocation concealment. Only 8 trials $[38,47,53,59-61,66$, 69] have mentioned adequate blinding of participants and personnel while the remaining studies were unclear. The item for evaluating the outcome was shown a relative completeness, except these trials $[36,37,39,70,71]$. Three trials were judged as high risk of bias based on other bias items [37, 41, 


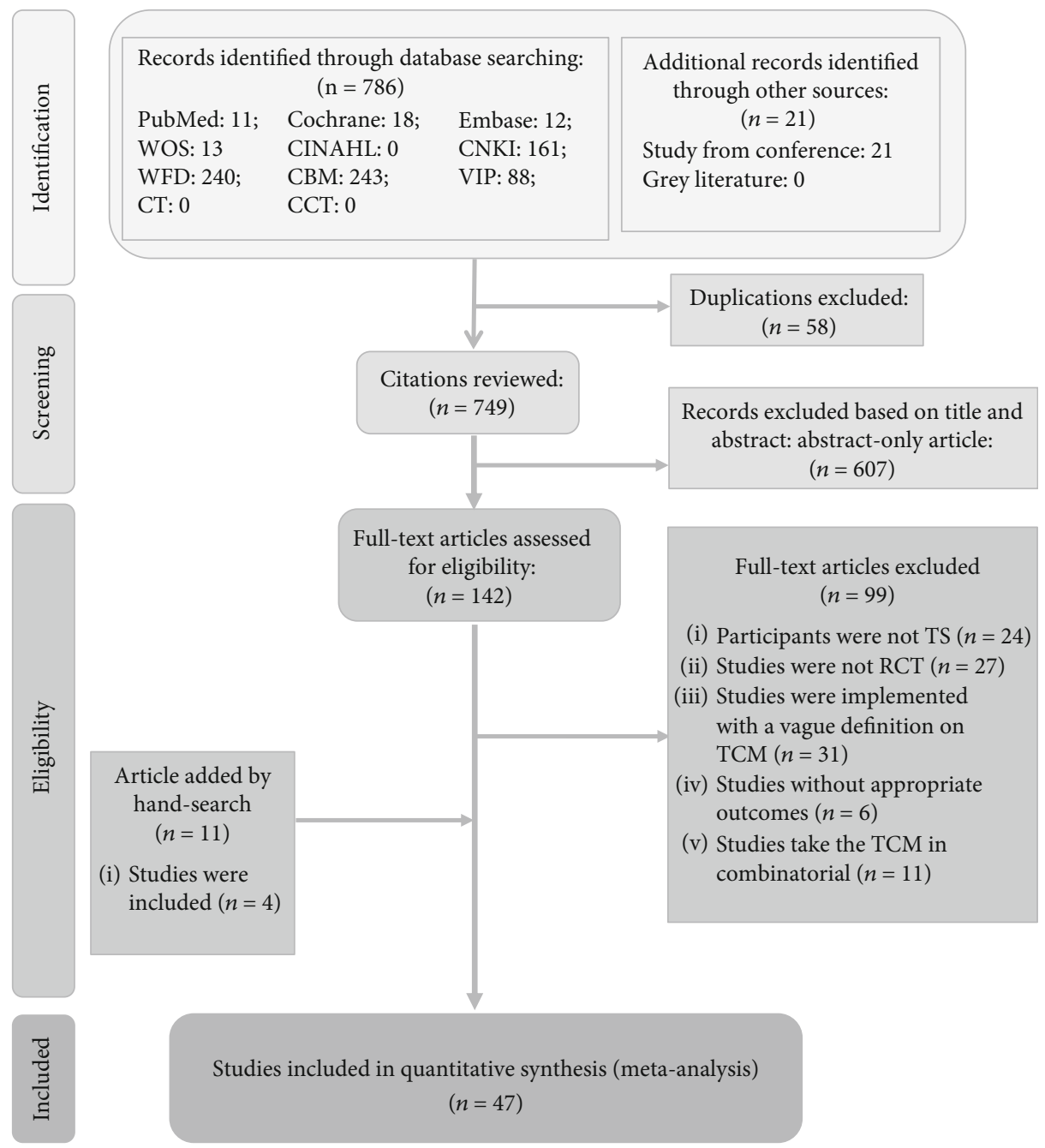

FIGURE 1: Literature review flowchart. CBM = Chinese Biomedical Literature Database; CCT = Chinese Clinical Trial Registry; CNKI = China National Knowledge Infrastructure database; $\mathrm{CT}=$ clinical trials; RCT = randomized controlled trial; TS = Tourette Syndrome; VIP = Chinese Scientific Journal database; WFD = Wanfang database; WOS = Web of Science.

62]. Overall and individual quality was detailed in Supplement Figure 1 and Supplement Figure 2.

\subsection{Primary Outcome}

3.3.1. Clinical Efficacy. All 47 trials investigated the relationship of response rate between TCM and CG, and our results showed that TCM was more likely to take effect than the CG in clinical efficacy with a statistical significance OR of 1.15 $\left(\mathrm{OR}=1.15,95 \% \mathrm{CI}: 1.06\right.$ to $\left.1.26, I^{2}=0.00 \%\right)$ (shown in Table 2). The funnel plot hint of no publication bias was observed (Supplement Figure 3), and equal indication was found in the quantitative egger test $(P$ value $=0.007)$ (shown in Supplement Figure 4).

\subsection{Secondary Outcome}

3.4.1. Yale Global Tic Severity Scale. There were 26 studies concentrated on YGTSS total scores. Results presented that patients who received TCM were notably improving their YGTSS total scores when compared with the CG
$\left(\mathrm{SMD}=-0.21,95 \% \mathrm{CI}:-0.29\right.$ to $\left.-0.14, I^{2}=12.30 \%\right)$ (shown in Table 2).

In terms of the YGTSS motor tic scores, an item of YGTSS, 9 studies targeted at it and the pooled results reported remarkable improvement of the TCM group compared with the CG $((\mathrm{OR}=1.26,95 \% \mathrm{CI}$ : 1.002 to 1.58 , $\left.I^{2}=0.00 \%\right)$ and $(\mathrm{SMD}=-0.37,95 \% \mathrm{CI}:-0.55$ to -0.19 , $\left.I^{2}=41.50 \%\right)$ ). Likewise, results from 7 studies revealed that patients with TS in the TCM group got a significant improvement in YGTSS vocal tic scores (SMD $=-0.23,95 \%$ CI: -0.35 to $-0.10, I^{2}=0.00 \%$ ), but a nonsignificant improved performance was detected while the ES was accounted in a dichotomous variable $\left(\mathrm{OR}=1.17,95 \% \mathrm{CI}: 0.92\right.$ to $\left.1.49, I^{2}=0.00 \%\right)$ (shown in Table 2).

3.4.2. Traditional Chinese Medicine Syndrome Score Scale. 14 and 6 studies recorded the endpoints of TCMSSS with a dichotomous variable and numerical variable, respectively. Among included studies with different variable types, metaanalysis results showed that compared with the CG, the 
TABle 1: Demographic characteristics of literatures.

\begin{tabular}{|c|c|c|c|c|c|c|c|c|c|}
\hline \multirow{2}{*}{ Publication } & \multicolumn{2}{|c|}{ Sample size } & \multirow{2}{*}{$\begin{array}{c}\text { Proportion of } \\
\text { male }(\%)\end{array}$} & \multicolumn{2}{|c|}{ Age mean $\pm \mathrm{SD} /$ range } & \multirow{2}{*}{ Diagnose } & \multirow{2}{*}{$\begin{array}{c}\text { Therapeutic } \\
\text { course }\end{array}$} & \multirow{2}{*}{ Outcome } & \multirow{2}{*}{$\mathrm{ROB}$} \\
\hline & TCM & CG & & TCM & $\mathrm{CG}$ & & & & \\
\hline Wenzhong Zhang, 2013 & 30 & 20 & $86.00 \%$ & \multicolumn{2}{|c|}{$6-14$} & CCMD-III & $6 \mathrm{Ws}$ & (1) (5) & $\mathrm{U}$ \\
\hline Weibin Gao, 2007 & 60 & 60 & $71.66 \%$ & \multicolumn{2}{|c|}{$4-21$} & CCMD-2-R & $3 \mathrm{Ws}$ & (1)(2)(3)(4) & $\mathrm{U}$ \\
\hline Dahua Wu, 2007 & 22 & 22 & $75.00 \%$ & $9.66 \pm 2.83$ & $10.03 \pm 3.08$ & DSM-IV & $4 \mathrm{Ws}$ & (1)(2) 6) & $\mathrm{L}$ \\
\hline Guolan Ge, 2013 & 31 & 31 & $79.03 \%$ & \multicolumn{2}{|c|}{$6.08 \pm 2.05$} & DSM-IV & $12 \mathrm{Ws}$ & (1) & $\mathrm{H}$ \\
\hline Linghua Deng, 2014 & 30 & 30 & $70.00 \%$ & $8.09 \pm 1.74$ & $8.47 \pm 1.78$ & DSM-IV & $8 \mathrm{Ws}$ & (1)(2)(6) & $\mathrm{H}$ \\
\hline Congling Sun, 2008 & 33 & 33 & $78.79 \%$ & \multicolumn{2}{|c|}{$6-18$} & DSM-IV & $24 \mathrm{Ws}$ & (1)(3)(4)6 & $\mathrm{L}$ \\
\hline Anyuan Li, 2008 & 60 & 60 & $71.67 \%$ & \multicolumn{2}{|c|}{$3-25$} & DSM-IV & $24 \mathrm{Ws}$ & (1)(3)(4)(7) & $\mathrm{L}$ \\
\hline Ruiping Ma, 2006 & 60 & 60 & $71.67 \%$ & $10.26 \pm 4.05$ & $10.98 \pm 3.80$ & DSM-IV & $24 \mathrm{Ws}$ & (1)(3)(4)(7) & $\mathrm{L}$ \\
\hline Anyuan Li, 2013 & 42 & 43 & $70.59 \%$ & $10.48 \pm 2.85$ & $10.53 \pm 2.78$ & DSM-IV & $24 \mathrm{Ws}$ & (1)(2)(3)(4)(6)(7) & $\mathrm{L}$ \\
\hline Riming Wu, 2004 & 30 & 30 & $78.33 \%$ & \multicolumn{2}{|c|}{$4-12$} & CCMD-2-R & $8 \mathrm{Ws}$ & (1) 7 & $\mathrm{U}$ \\
\hline Ying Tang, 2015 & 50 & 50 & $51.00 \%$ & $8.60 \pm 3.20$ & $8.30 \pm 3.40$ & Other & $12 \mathrm{Ws}$ & (1) (7) & $\mathrm{U}$ \\
\hline Hengping Chen, 2009 & 43 & 30 & $27.40 \%$ & $9.20 \pm 0.80$ & $9.10 \pm 0.90$ & CCMD-2-R & $12 \mathrm{Ws}$ & (1) (7) & $\mathrm{U}$ \\
\hline Guiping Li, 2013 & 54 & 54 & $53.70 \%$ & $6.50 \pm 3.30$ & $7.20 \pm 3.80$ & CCMD-2-R & $8 \mathrm{Ws}$ & (1) (2) & $\mathrm{U}$ \\
\hline Xiubo Du, 2011 & 32 & 30 & $67.74 \%$ & $7.92 \pm 3.59$ & $8.09 \pm 3.43$ & DSM-IV & $12 \mathrm{Ws}$ & (1) (7) & $\mathrm{U}$ \\
\hline Yan Liu, 2009 & 60 & 40 & $58.00 \%$ & $10.26 \pm 4.05$ & $10.98 \pm 3.80$ & DSM-IV & $24 \mathrm{Ws}$ & (1) (3)(4) (7) & $\mathrm{U}$ \\
\hline Bo wang, 2013 & 48 & 48 & $71.88 \%$ & $13.40 \pm 4.20$ & $12.90 \pm 3.90$ & DSM-IV & $12 \mathrm{Ws}$ & (1) (2) & $\mathrm{U}$ \\
\hline Lijun Deng, 2015 & 40 & 40 & $76.25 \%$ & $9.14 \pm 2.21$ & $9.16 \pm 2.19$ & DSM-IV & $12 \mathrm{Ws}$ & (1)(2) (6) & $\mathrm{L}$ \\
\hline Feifei Chen, 2011 & 30 & 30 & $56.67 \%$ & $8.00 \pm 2.36$ & $9.00 \pm 2.65$ & DSM-IV & $12 \mathrm{Ws}$ & (1) (2) & $\mathrm{L}$ \\
\hline Tingting Fu, 2007 & 33 & 33 & $71.21 \%$ & $7.97 \pm 2.79$ & $8.29 \pm 2.73$ & DSM-IV & $24 \mathrm{Ws}$ & (1)(2)(3)(4)(6)(7) & $\mathrm{L}$ \\
\hline Feng Han, 2015 & 30 & 30 & $75.00 \%$ & $8.94 \pm 2.18$ & $9.15 \pm 2.27$ & DSM-IV-R & $8 \mathrm{Ws}$ & (1) (6) & $\mathrm{U}$ \\
\hline Huawei Li, 2011 & 36 & 36 & $45.83 \%$ & $9.55 \pm 2.91$ & $9.20 \pm 3.02$ & CCMD-III & $8 \mathrm{Ws}$ & (1)(2) (6) & $\mathrm{U}$ \\
\hline Rongyi Zhou, 2016 & 60 & 60 & $78.33 \%$ & $5-15$ & $5-16$ & Other & $16 \mathrm{Ws}$ & (1) (7) & $\mathrm{H}$ \\
\hline Siyuan Hu, 2014 & 328 & 110 & $80.37 \%$ & $9.86 \pm 2.92$ & $9.35 \pm 2.94$ & Other & 4Ws & (1)(2)(6) (7) & $\mathrm{L}$ \\
\hline Qi Sun, 2016 & 36 & 36 & $56.00 \%$ & \multicolumn{2}{|c|}{$4-35$} & Other & $8 \mathrm{Ws}$ & (1) 7 & $\mathrm{H}$ \\
\hline Lifeng Shi, 2009 & 30 & 30 & $70.00 \%$ & \multicolumn{2}{|c|}{$5-18$} & DSM-IV & $8 \mathrm{Ws}$ & (1) (7) & $\mathrm{H}$ \\
\hline Yi Zheng,2016 & 362 & 116 & $84.52 \%$ & $9.60 \pm 3.10$ & $9.90 \pm 2.80$ & DSM-IV & $8 \mathrm{Ws}$ & (1)(6)(7) & $\mathrm{L}$ \\
\hline Jinhui Li, 2016 & 118 & 115 & $54.35 \%$ & \multicolumn{2}{|c|}{ 4-14 } & DSM-IV & $6 \mathrm{Ws}$ & (1)(2)(6) & $\mathrm{L}$ \\
\hline Xingyou Zhao, 2007 & 30 & 30 & $53.33 \%$ & \multicolumn{2}{|c|}{$4-18$} & DSM-IV & $12 \mathrm{Ws}$ & (1)(2)(3)(4)(6)(7) & $\mathrm{L}$ \\
\hline Na Yang, 2016 & 353 & 118 & $81.85 \%$ & \multicolumn{2}{|c|}{ NR } & CCMD-III & $6 \mathrm{Ws}$ & (1)(2)67) & $\mathrm{L}$ \\
\hline Rong Ma, 2010 & 336 & 113 & NR & \multicolumn{2}{|c|}{$4-18$} & DSM-IV & $6 \mathrm{Ws}$ & (1) (2) (3) (4)(7) & $\mathrm{H}$ \\
\hline Zheng Hong, 2015 & 43 & 42 & $84.71 \%$ & $7.84 \pm 1.79$ & $8.31 \pm 1.98$ & DSM-IV-R & $12 \mathrm{Ws}$ & (1)(2)(5)(6)(7) & $\mathrm{U}$ \\
\hline Meiying Liu, 2011 & 30 & 30 & $75.00 \%$ & $8.17 \pm 2.87$ & $9.20 \pm 3.16$ & $\begin{array}{c}\text { DSM-IV- } \\
\text { TR }\end{array}$ & $8 \mathrm{Ws}$ & (1)(2)(3)(4)(5)(6)(7) & $\mathrm{L}$ \\
\hline Jin Li, 2010 & 106 & 105 & NR & \multicolumn{2}{|c|}{$4-18$} & CCMD-III & $4 \mathrm{Ws}$ & (1)(2)(3)(4)(5)(6)(7) & $\mathrm{L}$ \\
\hline Haiying Wei, 2013 & 30 & 30 & $78.33 \%$ & $8.53 \pm 2.49$ & $8.33 \pm 2.48$ & CCMD-III & $8 \mathrm{Ws}$ & (1)(2)(6) 7 & $\mathrm{U}$ \\
\hline Di Zhang, 2013 & 30 & 30 & $73.33 \%$ & \multicolumn{2}{|c|}{ NR } & CCMD-III & $6 \mathrm{Ws}$ & (1)(2)(6) 7 & $\mathrm{U}$ \\
\hline Chuang Zhao, 2014 & 30 & 30 & $70.00 \%$ & $8.57 \pm 2.52$ & $8.23 \pm 2.45$ & Other & $8 \mathrm{Ws}$ & (1)(3)(4) & $\mathrm{U}$ \\
\hline L Zhao, 2010 & 33 & 31 & $89.06 \%$ & $11.95 \pm 2.93$ & $12.50 \pm 2.87$ & $\begin{array}{c}\text { DSM-IV- } \\
\text { TR }\end{array}$ & $8 \mathrm{Ws}$ & (1)(2)(3)(4) & $\mathrm{L}$ \\
\hline Anyuan Li, 2009 & 60 & 30 & $57.78 \%$ & $9.59 \pm 3.00$ & $9.60 \pm 2.95$ & DSM-IV & $24 \mathrm{Ws}$ & (1)(2)(3)(4)(5) (7) & $\mathrm{L}$ \\
\hline Min Wu, 2010 & 31 & 30 & $83.61 \%$ & $8.61 \pm 3.16$ & $9.30 \pm 2.32$ & ICD-10 & $24 \mathrm{Ws}$ & (1) (2) & $\mathrm{L}$ \\
\hline Min Wu, 2009 & 41 & 40 & $81.48 \%$ & $9.70 \pm 2.01$ & $9.10 \pm 1.13$ & DSM-IV & $24 \mathrm{Ws}$ & (1)(2) & $\mathrm{L}$ \\
\hline Yunchou Wu,2015 & 32 & 32 & $70.31 \%$ & 5 & & DSM-IV & $6 \mathrm{Ws}$ & (1)(6)(7) & $\mathrm{U}$ \\
\hline Feng Yang, 2012 & 30 & 30 & $61.67 \%$ & 7.5 & 7.3 & DSM-IV & $4 \mathrm{Ws}$ & (1)(5) & $\mathrm{H}$ \\
\hline Fen Wang, 2011 & 30 & 30 & $76.67 \%$ & $4-$ & & Other & $4 \mathrm{Ws}$ & (1) (7) & $\mathrm{U}$ \\
\hline
\end{tabular}


TABLE 1: Continued.

\begin{tabular}{|c|c|c|c|c|c|c|c|c|c|}
\hline \multirow{2}{*}{ Publication } & \multicolumn{2}{|c|}{ Sample size } & \multirow{2}{*}{$\begin{array}{c}\text { Proportion of } \\
\text { male }(\%)\end{array}$} & \multicolumn{2}{|c|}{ Age mean $\pm \mathrm{SD} /$ range } & \multirow{2}{*}{ Diagnose } & \multirow{2}{*}{$\begin{array}{c}\text { Therapeutic } \\
\text { course }\end{array}$} & \multirow{2}{*}{ Outcome } & \multirow{2}{*}{$\mathrm{ROB}$} \\
\hline & TCM & CG & & TCM & $\mathrm{CG}$ & & & & \\
\hline Xinhui Shan, 2016 & 45 & 45 & $81.11 \%$ & $8.00 \pm 2.5$ & $9.00 \pm 2.7$ & Other & $12 \mathrm{Ws}$ & (1)(2)(5)(7) & $\mathrm{U}$ \\
\hline Jiaomei Feng, 2011 & 33 & 33 & $71.21 \%$ & $8.48 \pm 2.50$ & $8.18 \pm 2.57$ & DSM-IV & $12 \mathrm{Ws}$ & (1)(2)(3)(4)(5)(6)(7) & $\mathrm{U}$ \\
\hline Jinping Fan, 2017 & 60 & 60 & $64.17 \%$ & $10.33 \pm 3.14$ & $10.90 \pm 3.54$ & DSM-V & $12 \mathrm{Ws}$ & (1)(2)(6) (7) & $\mathrm{U}$ \\
\hline Jingyu Qiu, 2010 & 60 & 40 & $58.00 \%$ & $11.03 \pm 4.05$ & $11.09 \pm 3.80$ & DSM-IV & $24 \mathrm{Ws}$ & (1)(3)(4)(7) & $\mathrm{U}$ \\
\hline
\end{tabular}

CCMD: Chinese Classification of Mental Disorders; CG: control group; DSM: the Diagnostic and Statistical Manual; H: high; L: Low; U: unclear; NR: not reported; ROB: risk of bias; TCM: traditional Chinese medicine; outcome: (1) clinical efficacy; (2) YGTSS (Yale Global Tic Severity Scale); (3) YGTSS motor tic scores; (4) YGTSS vocal tic scores; (5) recurrence rate; (6) TCMSSS (TCM Syndrome Score Scale); (7) adverse reaction.

TCM group had a conspicuous improvement $((\mathrm{OR}=1.20$, 95\% CI: 1.04 to $\left.1.37, I^{2}=0.00 \%\right)$ and (SMD $=-0.69,95 \%$ CI: -1.17 to $\left.-0.21, I^{2}=80.50 \%\right)$ ) (shown in Table 2 ).

3.4.3. Recurrence Rate and Adverse Reaction. Six studies used recurrence rate as one of their endpoints. Furthermore, there were 29 (studies) collecting adverse reaction as their endpoints. When it comes to recurrence rate, the TCM group marked a higher likelihood to obviously contain the recurrence $\left(\mathrm{OR}=0.44,95 \% \mathrm{CI}: 0.24\right.$ to $\left.0.78, I^{2}=0.00 \%\right)$. As for adverse reaction, combined data regarding the TCM group was prominent in reducing the adverse effect $(\mathrm{OR}=0.32$, 95\% CI: 0.24 to $0.43, I^{2}=32.90 \%$ ) (shown in Table 2).

$I^{2}$ values reflected that there existed mild magnitude of heterogeneity across the included studies based on the outcome of YGTSS motor tic scores and adverse reaction, and severe heterogeneity in TCMSSS (shown in Table 2).

3.5. Subgroup Analyses. The prespecified subgroup analyses are eventually shown in Table 2 , which was divided into 8 categories. The overwhelming majority of subgroup analyses yielded consistent results, i.e., items under the subgroup were of statistical significance (illness course group, (average course $\leq 3$ years, $\mathrm{OR}=1.14,95 \% \mathrm{CI}: 0.99$ to 1.30 ) vs. (average course $>3$ years, $\mathrm{OR}=1.15, \mathrm{CI}: 0.93$ to 1.42 ); condition of the disease group, (mild, $\mathrm{OR}=1.06,95 \% \mathrm{CI}$ : 0.71 to 1.61 ) vs. (moderate-severe, $\mathrm{OR}=1.09,95 \% \mathrm{CI}$ : 0.80 to 1.48 ); diagnostic standards, (CCMD-II/III, OR $=1.18,95 \%$ CI: 1.00 to 1.39 ) vs. (DSM-IV, OR =1.15, 95\% CI:1.09 to 1.29); risk of bias group, (high risk of bias, $\mathrm{OR}=1.15,95 \% \mathrm{CI}$ : 1.06 to 1.26 ) vs. (low and unclear risk of bias, OR $=1.16,95 \% \mathrm{CI}: 1.06$ to 1.28)); however, when the publication years were taken into consideration, years later than 2010 (OR $=1.18, \mathrm{CI}$ : 1.06 to 1.32) saw a notable improvement compared with the CG while the remaining (cases) whose publication years were no later than 2010 had little improvement by detection $(\mathrm{OR}=1.10, \mathrm{CI}: 0.96$ to 1.27$)$. In terms of the total sample size group, the sample size $\leq 100(\mathrm{OR}=1.11, \mathrm{CI}: 0.98$ to 1.26$)$ showed an inconsistency with the sample size $>100$ $(\mathrm{OR}=1.19, \mathrm{CI}: 1.05$ to 1.34$)$ that the former experienced a remarkable improvement of TS patients. Finally, the results in regions with low or middle income $(\mathrm{OR}=1.18, \mathrm{CI}: 1.06$ to 1.32 ) were prominently superior to the $\mathrm{CG}$ and regions with high income $(\mathrm{OR}=1.11, \mathrm{CI}: 0.97$ to 1.27$)$.

\section{Discussion}

From the overall results of our study involving 47 RCTs and 5437 patients, compared with the control group, TS patients assigned to the TCM group witnessed a higher possibility of more effective treatment in clinical efficacy $(\mathrm{OR}=1.15,95 \%$ CI: 1.06 to 1.26 ), YGTSS total scores (SMD $=-0.21,95 \%$ CI: -0.29 to -0.14$)$, TCMSSS (OR $=1.20,95 \% \mathrm{CI}: 1.04$ to 1.37), recurrence rate $(\mathrm{OR}=0.44,95 \% \mathrm{CI}: 0.24$ to 0.78$)$, and adverse reaction ( $\mathrm{OR}=0.32,95 \% \mathrm{CI}: 0.24$ to 0.43 ), while it remained to be seen whether TCM could improve YGTSS vocal tic scores of TS patients $(\mathrm{OR}=1.17,95 \% \mathrm{CI}$ : 0.92 to 1.49). The summarized findings above suggested that TCM might achieve a relative effectiveness to alleviate the clinical symptoms of TS patients with a low risk of adverse reactions.

A high proportion of 47 included studies set their control group as the Western medicine (WM) of antipsychotics such as haloperidol and tiapride, which have been recommended by The European Society for the Study of Tourette Syndrome (ESSTS) as the medications to treat TS patients [73, 74]. However, pooled results from our study indicated that the intervention group of TCM was apparently more effective than WM in clinical efficacy rate or adverse effect. Chinese medicine categorizes TS into three types, namely, convulsion, muscular twitch, and cramp [44]. Most TS patients would experience symptoms like repetitive respiratory tract infection, nonrhythmic tics, which are caused by "wind invasion" into the lung - a "lung treatment" theory in TCM, accounting for the streptococcal infection, whose common symptoms are fever, stuffy nose, and sore throat [45]. Based on the visceral function of Chinese medicine, in order to improve the symptoms of TS patients, TCM often uses a combination of multiple Chinese herbals as their prescription to alleviate the wind and drive the chill out of the mind to maintain body homoeostasis [44]. Ningdong granule is regarded as a widespread Chinese prescription consisting of eight Chinese herbals with the function of antispasm, antiepilepsy, and sedation. It can further take effect as a nonspecific immune role with various degrees to alleviate the conditions of spasms and convulsions by inhibiting the hemolytic streptococcus and Streptococcus viridans [44]. As the authoritative scale for measuring the clinical effectiveness of TS patients, the total scores of YGTSS meant an obvious reduction in the TCM group which was consistent with the previous studies even in insufficient trials included [17]. 
TABLE 2: Primary results based on various outcomes and subgroup analyses.

\begin{tabular}{|c|c|c|c|c|c|}
\hline \multirow{2}{*}{ Meta-analyses variables } & \multirow{2}{*}{ No. of studies } & \multicolumn{2}{|c|}{ No. of patients } & \multirow{2}{*}{ Pool effect size } & \multirow{2}{*}{$I^{2}$} \\
\hline & & TCM & CG & & \\
\hline \multicolumn{4}{|l|}{ Numerical variable } & \multicolumn{2}{|l|}{ Pooled ORs (95\% CI) } \\
\hline Clinical efficacy & 47 & 3231 & 2206 & 1.15 (1.06 to 1.26$)$ & $0.00 \%$ \\
\hline YGTSS motor tic scores & 9 & 396 & 356 & $1.26(1.00$ to 1.58$)$ & $0.00 \%$ \\
\hline YGTSS vocal tic scores & 9 & 396 & 356 & $1.17(0.92$ to 1.49$)$ & $0.00 \%$ \\
\hline Recurrence rate & 6 & 241 & 200 & $0.44(0.24$ to 0.78$)$ & $0.00 \%$ \\
\hline TCMSSS & 14 & 1265 & 807 & $1.20(1.04$ to 1.37$)$ & $0.00 \%$ \\
\hline Adverse reaction & 29 & 2504 & 1496 & $0.32(0.24$ to 0.43$)$ & $32.90 \%$ \\
\hline \multicolumn{4}{|l|}{ Continuous variable } & \multicolumn{2}{|l|}{ Pooled SMDs (95\% CI) } \\
\hline YGTSS total scores & 26 & 2401 & 1441 & $-0.21(-0.29$ to -0.14$)$ & $12.30 \%$ \\
\hline YGTSS motor tic scores & 7 & 670 & 415 & $-0.37(-0.55$ to -0.19$)$ & $41.50 \%$ \\
\hline YGTSS vocal tic scores & 7 & 670 & 415 & $-0.23(-0.35$ to -0.10$)$ & $0.00 \%$ \\
\hline TCMSSS & 6 & 194 & 195 & $-0.69(-1.17$ to -0.21$)$ & $80.50 \%$ \\
\hline \multicolumn{4}{|c|}{ Subgroup analysis based on the outcome of YGTSS } & \multicolumn{2}{|l|}{ Pooled SMDs (95\% CI) } \\
\hline \multicolumn{6}{|c|}{ Disease course } \\
\hline Overall & 33 & 1653 & 1308 & $1.14(1.02$ to 1.28$)$ & $0.00 \%$ \\
\hline Average course $\leq 3 \mathrm{Ys}$ & 24 & 1198 & 936 & $1.14(0.99$ to 1.30$)$ & $0.00 \%$ \\
\hline Average course $>3 \mathrm{Ys}$ & 9 & 455 & 372 & $1.15(0.93$ to 1.42$)$ & $0.00 \%$ \\
\hline \multicolumn{6}{|l|}{ Condition of disease } \\
\hline Overall & 9 & 299 & 268 & $1.08(0.84$ to 1.38$)$ & $0.00 \%$ \\
\hline Mild & 3 & 121 & 90 & $1.06(0.71$ to 1.61$)$ & $0.00 \%$ \\
\hline Moderate-severe & 6 & 178 & 178 & $1.09(0.80$ to 1.48$)$ & $0.00 \%$ \\
\hline \multicolumn{6}{|l|}{ Publication year } \\
\hline Overall & 47 & 3231 & 2206 & 1.15 (1.06 to 1.26$)$ & $0.00 \%$ \\
\hline$\leq 2010$ & 18 & 1128 & 817 & $1.10(0.96$ to 1.27$)$ & $0.00 \%$ \\
\hline$>2010$ & 29 & 2103 & 1389 & $1.18(1.06$ to 1.32$)$ & $0.00 \%$ \\
\hline \multicolumn{6}{|l|}{ Therapeutic course } \\
\hline Overall & 47 & 3231 & 2206 & 1.15 (1.06 to 1.26$)$ & $0.00 \%$ \\
\hline$\leq 12 \mathrm{Ws}$ & 24 & 2206 & 1268 & $1.18(1.05$ to 1.32$)$ & $0.00 \%$ \\
\hline$>12 \mathrm{Ws}$ & 23 & 1025 & 938 & $1.12(0.96$ to 1.28$)$ & $0.00 \%$ \\
\hline \multicolumn{6}{|l|}{ Diagnostic standards } \\
\hline Overall & 47 & 3231 & 2206 & 1.15 (1.06 to 1.26$)$ & $0.00 \%$ \\
\hline CCMD & 11 & 1100 & 623 & $1.18(1.00$ to 1.39$)$ & $4.30 \%$ \\
\hline DSM & 29 & 1849 & 1302 & 1.15 (1.09 to 1.29$)$ & $0.00 \%$ \\
\hline Other & 7 & 282 & 281 & $1.10(0.85$ to 1.41$)$ & $0.00 \%$ \\
\hline \multicolumn{6}{|l|}{ Total sample size } \\
\hline Overall & 47 & 3231 & 2206 & 1.15 (1.06 to 1.26$)$ & $0.00 \%$ \\
\hline$\leq 100$ & 32 & 1104 & 1045 & $1.11(0.98$ to 1.26$)$ & $0.00 \%$ \\
\hline$>100$ & 15 & 2127 & 1161 & $1.19(1.05$ to 1.34$)$ & $6.30 \%$ \\
\hline \multicolumn{6}{|l|}{ Region } \\
\hline Overall & 47 & 3231 & 2206 & 1.15 (1.06 to 1.26$)$ & $0.00 \%$ \\
\hline High income & 26 & 2078 & 1053 & $1.11(0.97$ to 1.27$)$ & $0.00 \%$ \\
\hline Low and middle income & 21 & 1153 & 1153 & 1.18 (1.06 to 1.32$)$ & $0.00 \%$ \\
\hline \multicolumn{6}{|l|}{ ROB quality } \\
\hline Overall & 47 & 3231 & 2206 & 1.15 (1.06 to 1.26$)$ & $0.00 \%$ \\
\hline High & 7 & 989 & 945 & 1.15 (1.06 to 1.26$)$ & $0.00 \%$ \\
\hline Low/unclear & 40 & 2242 & 1261 & $1.16(1.06$ to 1.28$)$ & $0.00 \%$ \\
\hline
\end{tabular}

CI: confidence interval; CCMD: Chinese Classification of Mental Disorders; CG: control group; DSM: the Diagnostic and Statistical Manual; OR: odds ratio; ROB: risk of bias; SMD: standard mean differences; TCM: traditional Chinese medicine; TCMSSS: TCM Syndrome Score Scale; YGTSS: Yale Global Tic Severity Scale. 
Our main findings reinforce the existing evidence that TCM was more inclined to function in the treatment of tic symptom.

Not only was the superiority reflected in its effectiveness but also it was endorsed by a stable adverse effect. TS patients treated with WM (haloperidol, risperidone, etc.) were more prone to suffer from dizziness, drowsiness, and constipation even the extrapyramidal system reaction easily. Nonetheless, the adverse reactions that occurred among patients who received TCM were less frequently observed than those observed in WM [69, 75]. Furthermore, TCM, with its advantages of efficacy in both the short term and the long term, proved itself worth introducing [44]. Apparently, as an essential component of Chinese medicine, acupuncture with its promising effectiveness seems to be as reputed as TCM and some articles indicated that the combination of acupuncture and TCM would optimize the effectiveness brought by the treatment $[76,77]$.

Based on the primary outcome, several significant differences were observed and introduced as follows. Compared with the studies with publication years no later than 2010 $(\mathrm{OR}=1.10, \mathrm{CI}: 0.96$ to 1.27$)$, studies with publication years over 2010 delivered a significant improvement in the effect of TCM $(\mathrm{OR}=1.18, \mathrm{CI}: 1.06$ to 1.32$)$. Among the total sample size item, there was greater improvement in the group with a relatively larger sample size $(N>100)(\mathrm{OR}=1.19$, CI: 1.05 to 1.34$)$ than the group with a smaller sample size $(N \leq 100)(\mathrm{OR}=1.11, \mathrm{CI}: 0.98$ to 1.26$)$. In addition, the subgroup that dealt with region diversity revealed that trials conducted in areas with relatively lower income $(\mathrm{OR}=1.18, \mathrm{CI}$ : 1.06 to 1.32 ) proved better improvement compared to that conducted in high-income areas $(\mathrm{OR}=1.11, \mathrm{CI}: 0.97$ to 1.27). All of the above results of the subgroup items may attribute to the design of studies, demographic characteristics of the participants, time line, level of the local economic development, and measurement problems of the research or the fidelity of execution among other issues.

An extensive search strategy was performed based on numerous databases to identify the optimal RCT regarding TS patients treated with TCM. Therefore, the number of included studies and their total sample size contributed directly to complete and robust results. Furthermore, the comprehensive outcomes in our study reliably presented the efficacy and safety of TCM with an approach of quantitative analysis.

Quite a few uncontrollable limitations might jeopardize the conclusion of our results. First, due to the application of TCM, all 47 trials were from China suggesting no original data from other regions in these studies. Moreover, all the included trials have not been registered in the relevant trial platform, which may directly affect the stability of our evidence. The second limitation lied in the quality of the 47 studies included in that $5[36,37,39,70,71]$ and $6[38,47$, $60,61,66,69]$ trials were rated as high risk of bias in incomplete outcome data and blinding of outcome assessment, respectively. Thirdly, although we implemented a quantitative analysis to ensure the rationality of different traditional Chinese medicine, heterogeneities ranging from mild to moderate were observed and special attention should be paid to the homogeneity question between them. Finally, the pres- ence of uncontrollable and uncertain biases, including selective reporting biases, unclear detection biases, and other biases (e.g., there existing mild to high heterogeneity between different interventions), is usually inevitable, which may be introduced in our primary analysis and potentially influence the quality of our results.

\section{Conclusion}

In a nutshell, we cautiously suggest that TCM may realize improvement for patients with TS in mitigating the symptoms and its safety may prove itself more acceptable than WM. More high-quality relevant RCTs need to be implemented for the establishment of a comprehensive trial basis in order that more evidence-based researches can be completed successfully.

\section{Conflicts of Interest}

The authors have no conflicts of interest to disclose.

\section{Authors' Contributions}

$\mathrm{Na}$ Wang and Dong-dong Qin contributed equally to this work and should be considered equivalent authors.

\section{Acknowledgments}

This study was supported by the National Natural Science Foundation of China (31960178 and 82074421), BK-Funds for the Transformation and Popularization of Scientific and Technological Achievements of Traditional Chinese Medicine (ID: [2019]386), Natural Science Foundation of Yunnan Province of China (2018FB053 and 2019FA007), Key-Area Research and Development Program of Guangdong Province (2019B030335001), China Postdoctoral Science Foundation (2018M631105), Yunnan Provincial Academician and Expert Workstation (202005AF150017 and 2019IC051), and Post-doctoral Training Program in Yunnan Province, BK-Innovation Team of Yunnan College of Traditional Chinese Medicine (ID: 2017 HC011).

\section{Supplementary Materials}

Supplementary 1. PubMed strategy.

Supplementary 2. Supplement Figure 1: risk of bias graph.

Supplementary 3. Supplement Figure 2: risk of bias summary.

Supplementary 4. Supplement Figure 3: funnel plots of clinical efficacy.

Supplementary 5. Supplement Figure 4: Egger plot of clinical efficacy.

\section{References}

[1] L. Scahill, R. H. Bitsko, S. N. Visser, and S. J. Blumberg, "Prevalence of diagnosed Tourette syndrome in persons aged 6-17 years - United States, 2007," Morbidity \& Mortality Weekly Report, vol. 58, no. 21, pp. 581-585, 2009. 
[2] M. M. Robertson, “A personal 35 year perspective on Gilles de la Tourette syndrome: assessment, investigations, and management," The Lancet Psychiatry, vol. 2, no. 1, pp. 88-104, 2015.

[3] M. H. Bloch and J. F. Leckman, "Clinical course of Tourette syndrome," Journal of Psychosomatic Research, vol. 67, no. 6, pp. 497-501, 2009.

[4] R. D. Freeman, D. K. Fast, L. Burd, J. Kerbeshian, and P. Sandor, "An international perspective on Tourette syndrome: selected findings from 3500 individuals in 22 countries," Developmental Medicine and Child Neurology, vol. 42, no. 7, pp. 436-447, 2000.

[5] I. Jalenques, F. Galland, L. Malet, D. Morand, and F. Durif, "Quality of life in adults with Gilles de la Tourette syndrome," BMC Psychiatry, vol. 12, 2012.

[6] E. A. Storch, L. J. Merlo, C. Lack et al., "Quality of life in youth with Tourette's syndrome and chronic tic disorder," Journal of Clinical Child and Adolescent Psychology, vol. 36, no. 2, pp. 217-227, 2007.

[7] E. F. Hedderick, C. M. Morris, and H. S. Singer, "Double-blind, crossover study of clonidine and levetiracetam in Tourette syndrome," Pediatric Neurology, vol. 40, no. 6, pp. 420-425, 2009.

[8] A. Meisel, C. Winter, R. Zschenderlein, and G. Arnold, "Tourette syndrome: efficient treatment with ziprasidone and normalization of body weight in a patient with excessive weight gain under tiapride," Movement Disorders, vol. 19, no. 8, pp. 991-992, 2004.

[9] C. M. Eddy, H. E. Rickards, and C. A. E. Review, "Treatment strategies for tics in Tourette syndrome," Therapeutic Advances in Neurological Disorders, vol. 4, no. 1, pp. 25-45, 2011.

[10] H. S. Singer, "Treatment of tics and Tourette syndrome," Current Treatment Options in Neurology, vol. 12, no. 6, pp. 539-561, 2010.

[11] J. Quezada and K. A. Coffman, "Current approaches and new developments in the pharmacological management of Tourette syndrome," CNS Drugs, vol. 32, no. 1, pp. 33-45, 2018.

[12] J. Jankovic and R. Kurlan, "Tourette syndrome: evolving concepts," Movement Disorders, vol. 26, no. 6, pp. 1149-1156, 2011.

[13] H. Y. Chen, Y. H. Lin, J. C. Wu et al., "Characteristics of pediatric traditional Chinese medicine users in Taiwan: a nationwide cohort study," Pediatrics, vol. 129, no. 6, pp. e1485-e1492, 2012.

[14] H. Yu, M. Han, W. Lin et al., "Efficacy of Chinese herbal injections for the treatment of primary Nephrotic syndrome: a Bayesian network meta-analysis of randomized controlled trials," Frontiers in Pharmacology, vol. 11, article 579241, 2020.

[15] X. Li and C. Zhang, "Comparative efficacy of nine antidepressants in treating Chinese patients with post-stroke depression: a network meta-analysis," Journal of Affective Disorders, vol. 266, pp. 540-548, 2020.

[16] X. Huang, X. Duan, Y. Zhu, K. Wang, J. Wu, and X. Tian, "Comparative efficacy of Chinese herbal injections for the treatment of community-acquired pneumonia: a Bayesian network meta-analysis of randomized controlled trials," Phytomedicine, vol. 63, article 153009, 2019.

[17] H. Qi, R. Liu, W. Zheng et al., "Efficacy and safety of traditional Chinese medicine for Tourette's syndrome: a meta-analysis of randomized controlled trials," Asian Journal of Psychiatry, vol. 47, article 101853, 2020.
[18] D. Moher, A. Liberati, J. Tetzlaff, D. G. Altman, and for the PRISMA Group, "Preferred reporting items for systematic reviews and meta-analyses: the PRISMA statement," $B M J$, vol. 339, article b2535, 2009.

[19] J. Higgins and S. Green, Cochrane Handbook for Systematic Reviews of Interventions, Version 5.1.0, The Cochrane Collaboration, 2013.

[20] Association AP, American Psychiatric Association: Diagnostic and Statistical Manual of Mental Disorders, American Psychiatric Association, 2000.

[21] J. F. Leckman, M. A. Riddle, M. T. Hardin et al., "The Yale Global Tic Severity Scale: initial testing of a clinician-rated scale of tic severity," Journal of the American Academy of Child and Adolescent Psychiatry, vol. 28, no. 4, pp. 566-573, 1989.

[22] J. Higgins and S. E. Green, "Cochrane handbook for systematic reviews of interventions version 5.1.0. The Cochrane Collaboration (Eds)," Naunyn-Schmiedebergs Archiv für Experimentelle Pathologie und Pharmakologie, vol. 5, no. 2, p. S38, 2011.

[23] J. P. T. Higgins and S. G. Thompson, "Quantifying heterogeneity in a meta-analysis," Statistics in Medicine, vol. 21, no. 11, pp. 1539-1558, 2002.

[24] V. Larry, "Advances in statistical methods for meta-analysis," New Directions for Program Evaluation, vol. 24, pp. 25-42, 1984.

[25] M. Egger, P. Jüni, C. Bartlett, F. Holenstein, and J. Sterne, "How important are comprehensive literature searches and the assessment of trial quality in systematic reviews? Empirical study," Health Technology Assessment, vol. 7, no. 1, pp. 1-82, 2003.

[26] W. Z. Zhang, "30 cases of Tourette's syndrome in children treated with cicada Xing Zhidong decoction," Jiangxi Journal of Traditional Chinese Medicine, vol. 44, no. 366, pp. 48-49, 2013.

[27] X. B. Du, “32 cases of children's multiple tic treated with Tianma Gouteng decoction and Zhijing powder," Traditional Chinese Medicine Research, vol. 24, no. 11, 2011.

[28] A. Y. Li, "Application of Ningdong granule in the treatment of multiple tic syndrome. Papers of the 30th academic conference of pediatric branch of Chinese society of traditional," Chinese Medicine, 2013.

[29] Y. Liu, Bunao Sedative Treatment of Children with Tourette's Syndrome Particles Clinical Research, Guangzhou University of traditional Chinese Medicine, 2009.

[30] J. P. Fan, "Clinical effect and mechanism of Xiaoeranshenbunao granule in the treatment of children with tic disorder Lishizhen medicine and materia medica research," vol. 28, no. 8, 2017.

[31] H. W. Li, "Clinical efficacy of Bupizhijing Tang on multiple tics," China Journal of Chinese Medicine, vol. 26, no. 160, 2011.

[32] F. F. Chen, The clinical evaluation of "zhichou pellet" in the treatment of Tourette syndrome of children, Hubei University of Traditional Chinese Medicine, 2011.

[33] G. P. Li, "Clinical observation of 54 children with Tourette syndrome treated by soothing the liver, activating collaterals and nourishing yin method," Journal of Luzhou Medical College, vol. 36, no. 5, 2013.

[34] F. Han, "Clinical observation of Chaihu Guizhi decoction in the treatment of multiple tic," Journal of Sichuan of Traditional Chinese Medicine, vol. 33, no. 4, 2015.

[35] H. Y. Wei, Clinical observation of granules of dousing the wind and stopping the hyperacivity in the treatment of children 
Touretee syndrome, Heilongjiang University of Traditional Chinese Medicine, 2013.

[36] L. H. Deng, Clinical observation of Jianpi Xifeng decoction in the treatment of children's multiple tic syndrome, Hunan University of traditional Chinese Medicine, 2014.

[37] R. Ma, "Clinical observation of Jintong-granules on treating tic disorder in children," Globale Traditional Chinese Medicine, vol. 3, no. 1, 2010.

[38] D. H. Wu, "The clinical observation of the therapeutic effect of qufenping on Tourette's syndrome," Chinese Journal of Information on Traditional Chinese Medicine, vol. 14, no. 7, 2007.

[39] L. F. Shi, Clinical observation of variation of Chaihujialonggumuli decoction on treating childhood multiple tic discorder, Heilongiiang University of traditional Chinese Medicine, 2009.

[40] H. P. Chen, "Clinical observation on 43 cases of children's tic treated by Qianzheng decoction," Zhejiang Journal of Traditional Chinese Medicine, vol. 44, no. 11, 2009.

[41] Q. Sun, "Clinical observation on Changma Xifeng tablets in the treatment of 36 cases of Tourette's syndrome," Management of Health Standards in China, vol. 131, no. 2, pp. 16749316, 2016.

[42] R. M. Wu, "Clinical observation on the treatment of 30 cases of children's multiple tic with Pinghua," Journal of Sichuan of Traditional Chinese Medicine, vol. 22, no. 7, 2004.

[43] X. H. Shan, "Clinical observation on the treatment of multiple tic with qiangzhi powder," Guangxi Journal of Traditional Chinese Medicine, vol. 39, no. 4, 2016.

[44] A. Li, S. Cong, H. Lu, J. Li, and L. Zhao, "Clinical observation on treatment of Tourette syndrome by integrative medicine," Chinese Journal of Integrative Medicine, vol. 15, no. 4, pp. 261-265, 2009.

[45] M. Wu, G. H. Xiao, J. M. Zhang et al., "Clinical research into Qufeng Zhidong recipe used to treat 31 children with tic disorder," Journal of Traditional Chinese Medicine, vol. 30, no. 3, pp. 163-170, 2010.

[46] B. Wang, "Clinical research of liver and kidney nourishing treatment for child tic disorder and its allergic reaction," Clinical Medicine Official Research, vol. 41, no. 5, 2013.

[47] J. Li, "Clinical research of Xifeng Zhidong tablet in treating multiple tics with liver wind and sputum stirring internally," Drugs \& Clinic, vol. 25, no. 2, 2010.

[48] C. Zhao, The clinical research on the treatment of Ziyin extinguish wind particles to yin deficiency pneumatic type of Tourett syndrome, Heilongjiang University of traditional Chinese Medicine, 2014.

[49] J. M. Feng, Clinical study and experimental study on treatment of Tourette's syndrome(TS) by Qiangzhisan, Shandong University of traditional Chinese Medicine, 2014.

[50] A. Y. Li, "Clinical study of Ningdong granule in the treatment of Tourette syndrome," Journal of Shandong University of Traditional Chinese Medicine, vol. 32, no. 1, 2008.

[51] J. Y. Qiu, “Clinical study on 60 cases of children's multiple tic treated by Xiaoer Anshen Bunao granule," Journal of Pediatric, vol. 16, no. 1, 2010.

[52] Z. Hong, "Clinical study on Shengqingjiangzhuo granules in treating Tourrette syndrome with syndrome of stirring wind due to phlegma-heat," China Journal of Chinese Medicine, vol. 30, no. 210, 2015.

[53] X. Y. Zhao, A clinical study on the treatment of children's multiple Tourette syndrome with qiangzidinghuofang, Shandong University of traditional Chinese Medicine, 2007.
[54] F. Wang, "Clinical study on the treatment of children's tic with cicada exuviate Gouteng decoction," Shandong Journal of traditional Chinese Medicine, vol. 30, no. 4, 2011.

[55] W. B. Gao, "A clinical study on the treatment of Tourette's syndrome with tongtongning," Journal Chinese Medicine and Pharmacy, vol. 35, no. 6, pp. 46-48, 2007.

[56] M. Y. Liu, Clinical study on treatment of Tourette syndrome(TS) by Tiqiziyin and Qingrezhenjing therapy, Shandong University of Traditional Chinese Medicine, 2011.

[57] C. L. Sun, Clinical Study on Treatment of Tourette's Syndrome (TS) by Liqijianpi and Xifenghuatan Therapy, Shandong University of Traditional Chinese Medicine, 2008.

[58] R. P. Ma, Clinical Study on Treatment of Tourettes Syndrome by Ningdong Granule, Shandong University, 2006.

[59] T. T. Fu, Clinical Study on Treatment of Tourette's Syndrome(TS) by Yishenqiangzhi Therapy, Shandong University of Traditional Chinese Medicine, 2007.

[60] J. H. Li, "Double blind randomized controlled trial of Jintong granule in the treatment of children with tic disorder," Journal of Traditional Chinese Medicine, vol. 57, no. 10, 2016.

[61] N. Yang, M. A. Rong, H. U. Si-Yuan et al., "Efficacy and safety of Choudongning capsule(CDN) in children with Tourette's syndrome of spleen deficiency and phlegm accumulation," China Journal of Chinese Materia Medica, vol. 41, no. 16, pp. 3100-3106, 2016.

[62] R. Y. Zhou, "Efficacy of Anshen Dingzhiling recipes in treating Tourette syndrome," Chinese Journal of Experimental Traditional Medical Formulae [zhongguo shi yan fang ji xue za zhi], vol. 22, no. 10, 2016.

[63] M. Wu, G.-h. Xiao, M. Yao et al., "Multicenter clinical study on the treatment of children's tic disorder with Qufeng Zhidong recipe," Chinese Journal of Integrative Medicine, vol. 15, no. 4, pp. 254-260, 2009.

[64] S. Y. Hu, "Phase III clinical research of Changma Xifeng tablets in treating multiple tics with liver wind and sputum stirring internally," Drugs \& Clinic, vol. 29, no. 9, 2015.

[65] Y. Tang, "PingganJianpi formula in the treatment of children's multiple tic-clinical observation of 50 cases," Journal of Traditional Chinese Medicine, vol. 26, no. 13, 2015.

[66] Y. Zheng, Z. J. Zhang, X. M. Han et al., "A proprietary herbal medicine (5-Ling granule) for Tourette syndrome: a randomized controlled trial," Journal of Child Psychology and Psychiatry, vol. 57, no. 1, pp. 74-83, 2016.

[67] D. Zhang, The quasi spleen and liver spread flat clinical observation of treatment of children with Tourette syndrome, Heilongjiang University of Traditional Chinese Medicine, 2013.

[68] Y. C. Wu, Research on Clinical Treatment with Changpuyujin Formula to Cure TS Caused by Wind and Phlegm, Gansu University of traditional Chinese Medicine, 2015.

[69] L. Zhao, A. Y. Li, H. Lv, F. Y. Liu, and F. H. Qi, “Traditional Chinese medicine Ningdong granule: the beneficial effects in Tourette's disorder," The Journal of International Medical Research, vol. 38, no. 1, pp. 169-175, 2010.

[70] G. L. Ge, "Treatment of 31 cases of multiple tic with deficiency of both heart and spleen by Guipi decoction," Chinese Journal of Practical Nervous Disease, vol. 16, no. 4, 2013.

[71] F. Yang, "Treatment of Tourette's syndrome in children with sudingjingxifeng decoction," Guangming Traditional Chinese Medicine, vol. 27, no. 9, 2012.

[72] L. J. Deng, "Znirouganzhidong decoction for the treatment of hyperactivity of liver and wind in children with multiple tic," 
Beijing Journal of Traditional Chinese Medicine, vol. 34, no. 5, 2015.

[73] the ESSTS Guidelines Group, V. Roessner, A. Plessen et al., "European clinical guidelines for Tourette syndrome and other tic disorders. Part II: pharmacological treatment," European Child \& Adolescent Psychiatry, vol. 20, no. 4, pp. 173-196, 2011.

[74] the ESSTS Guidelines Group, C. Verdellen, J. van de Griendt, A. Hartmann, and T. Murphy, "European clinical guidelines for Tourette syndrome and other tic disorders. Part III: behavioural and psychosocial interventions," European Child \& Adolescent Psychiatry, vol. 20, no. 4, pp. 197-207, 2011.

[75] S. Z. Wang, F. H. Qi, J. J. Li, L. Zhao, and A. Y. Li, "Effects of Chinese herbal medicine Ningdong granule on regulating dopamine (DA)/serotonin (5-TH) and gamma-amino butyric acid (GABA) in patients with Tourette syndrome," Bioscience Trends, vol. 6, no. 4, pp. 212-218, 2012.

[76] J. Yu, Y. Ye, J. Liu, Y. Wang, W. Peng, and Z. Liu, "Acupuncture for Tourette syndrome: a systematic review," EvidenceBased Complementary and Alternative Medicine, vol. 2016, Article ID 1834646, 10 pages, 2016.

[77] H. Zhang, "Observations on efficacy of acupuncture plus point injection for treatmentr of child twitch," Shanghai Journal of Acupuncture \& Moxibustion, vol. 24, no. 5, 2005. 